\title{
Hopf Bifurcation, Positively Invariant Set, and Physical Realization of a New Four-Dimensional Hyperchaotic Financial System
}

\author{
G. Kai, ${ }^{1,2}$ W. Zhang, ${ }^{1}$ Z. C. Wei, ${ }^{1,3}$ J. F. Wang, ${ }^{1}$ and A. Akgul ${ }^{4}$ \\ ${ }^{1}$ Beijing Key Laboratory of Nonlinear Vibrations and Strength of Mechanical Structures, College of Mechanical Engineering, \\ Beijing University of Technology, Beijing 100124, China \\ ${ }^{2}$ School of Mathematics and Statistics, Inner Mongolia University of Financial and Economics, Hohhot 100124, China \\ ${ }^{3}$ School of Mathematics and Physics, China University of Geosciences, Wuhan 430074, China \\ ${ }^{4}$ Department of Electrical and Electronics Engineering, Faculty of Technology, Sakarya University, 54187 Sakarya, Turkey
}

Correspondence should be addressed to W. Zhang; sandyzhang0@yahoo.com

Received 16 December 2016; Revised 10 February 2017; Accepted 9 March 2017; Published 10 April 2017

Academic Editor: Ahmad Taher Azar

Copyright @ 2017 G. Kai et al. This is an open access article distributed under the Creative Commons Attribution License, which permits unrestricted use, distribution, and reproduction in any medium, provided the original work is properly cited.

\begin{abstract}
This paper introduces a new four-dimensional hyperchaotic financial system on the basis of an established three-dimensional nonlinear financial system and a dynamic model by adding a controller term to consider the effect of control on the system. In terms of the proposed financial system, the sufficient conditions for nonexistence of chaotic and hyperchaotic behaviors are derived theoretically. Then, the solutions of equilibria are obtained. For each equilibrium, its stability and existence of Hopf bifurcation are validated. Based on corresponding first Lyapunov coefficient of each equilibrium, the analytical proof of the existence of periodic solutions is given. The ultimate bound and positively invariant set for the financial system are obtained and estimated. There exists a stable periodic solution obtained near the unstable equilibrium point. Finally, the dynamic behaviors of the new system are explored from theoretical analysis by using the bifurcation diagrams and phase portraits. Moreover, the hyperchaotic financial system has been simulated using a specially designed electronic circuit and viewed on an oscilloscope, thereby confirming the results of the numerical integrations and its real contribution to engineering.
\end{abstract}

\section{Introduction}

In recent years, economic dynamics become very prominent in the mainstream economics, and stochastic analysis is a popular way to interpret financial time series based on the existing information. Random exogenous shocks is usually considered to be the reason of a periodic behavior of the economic system by employing stochastic analyses, caused by factors outside the system. The shortcoming of the stochastic approach is incapable to illustrate the dynamics of financial systems [1].

An effective and rapid control method is very important for the government when some chaotic phenomenon appears [2-5]. A classical three-dimensional financial dynamic model describing the time variations of state adjustment has been reported [3], which includes the interest rate, the price index, and the investment demand. Later, another threedimensional financial chaotic risk dynamic system was constructed for management process of financial markets and proved to be controlled effectively [6]. Then Holyst and Urbanowicz [7] used the method of delayed feedback control (DFC) and proved that a chaotic financial system can be stabilized on various periodic orbits [8]. Reference [9] discussed the complex behaviors of a financial system with time-delayed feedback by numerical simulation. Normal form of a financial system with delaying has been derived, which is associated with Hopf and double Hopf bifurcations and makes the financial system more complicated $[10,11]$. In addition, the positively invariant sets of the dynamic system have a basic significance in the state constraints and control constraints, which are widely applied in different kinds of field: general 3-body problem [12], delay 
differential-equations [13], stability of dynamic system [14], robust attitude control schemes [15], relative motion control of the spacecraft [16], interconnected and time-delay systems [17], and permanent magnet synchronous motor system [18].

On the other hand, high dimensional control system is now a research hot topic, which has been applied in many fields. Zhang et al. introduced a three-dimensional ultimate bound and positively invariant set in which parameters are positive [19]. Wang et al. showed a smooth fourdimensional quadratic autonomous hyperchaotic system, which generated two novel double-wing periodic, quasiperiodic, and hyperchaotic attractors [20]. Wei and Zhang obtained the ultimate bound and positively invariant set is in a four-dimensional autonomous system [21]. Du et al. proposed hyperchaotic Rikitake system which was a novel four-dimensional autonomous nonlinear system and assured the existence of Hopf bifurcation [22]. A new fourdimensional quadratic autonomous hyperchaotic attractor was presented and the Hopf bifurcation at the equilibrium point was analyzed by Prakash and Balasubramaniam [23]. Therefore, the dynamical behaviors of hyperchaotic systems are more complex than chaotic systems and will have practical meanings for carrying out this research about financial systems [24]. Therefore, it is very necessary to explain complicated phenomena of financial dynamics by a thorough studying on the internal structural characteristics of hyperchaotic systems. On the viewpoint of mathematics and finance, these research points are attractive and rational. This paper constructs a new type of hyperchaotic financial system by nonlinear feedback method.

The remainder of the paper is organized as follows. Firstly, a new four-dimensional financial system is introduced by adding feedback controllers to the classic financial system in Section 2. And a sufficient condition for nonexistence of chaotic or hyperchaotic behaviors is obtained theoretically. Then the novel hyperchaotic financial system is confirmed numerically from Lyapunov exponents. Characterizations for the four-dimensional Hopf bifurcations are surveyed in Section 3. Section 4 introduces the ultimate bound and positively invariant set. The dynamic properties of the system are showed via bifurcation diagram in Section 5. In Section 6, a real contribution to engineering will be realized by an electronic circuit and oscilloscope in real time. Section 7 gives some conclusions.

\section{Financial System from Classic Financial Model}

2.1. Formulation of System. A financial dynamic system with different factors is reported in [3]. With the proper dimensions and appropriate coordinates, a simplified financial model is proposed:

$$
\begin{aligned}
& \dot{x}=z+(y-a) x, \\
& \dot{y}=1-b y-x^{2}, \\
& \dot{z}=-x-c z,
\end{aligned}
$$

where " $x$ " shows the interest rate that is defined as the price or cost of money for borrowing and return to lending. " $y$ " implies the investment demand, which will be directly affected by the interest rate. In addition, the supply and demand of the commercial goods and inflation rate cause changes of " $z$ "; let $a>0, b>0$, and $c>0$, respectively, be the saving amount, the cost per investment, and the elasticity of demand in commercial market.

This paper is dedicated to greatly survey the dynamical behaviors of a four-dimensional financial system which expanded from a known three-dimensional financial system and also proposes a simplified mathematical system. In this paper, we design the controlled system as follows:

$$
\begin{aligned}
& \dot{x}=z+(y-a) x, \\
& \dot{y}=1-b y-x^{2}, \\
& \dot{z}=-x-c z+u, \\
& \dot{u}=-d x y-k u-m z,
\end{aligned}
$$

where $u$ denotes control input and economically state intervention to balance the economic environment. For example, United States interest rates are determined by the Federal Reserve with considering short term economic targets. Regulatory agencies of open markets meet at certain intervals to monitor the economic and financial situation and decide on monetary policies. To decrease inflation and increase the purchasing power of the consumers, government will increase the interest rate. As a result of this, control input is the factor that interacts with all variables. This cross relation between interest rate, inflation (this also represents the price and goods and services which are denoted as " $z$ " in the study), and government regulations can be expressed from (2d), in which $d, k$, and $m$ mean corresponding amplitudes.

Therefore, it will be expected to study some complex dynamical behaviors about the proposed four-dimensional autonomous system (2a), (2b), (2c), and (2d).

2.2. Nonexistence of Chaotic or Hyperchaotic Behaviors. In addition, chaotic solutions do not exist for certain parameter values in system (2a), (2b), (2c), and (2d). We get three positive Lyapunov exponents, and it will be beneficial for us to find hyperchaos. More precisely, the main results are obtained as follows.

Theorem 1. A six-parameter family $(a, b, c, d, k, m)$ of system (2a), (2b), (2c), and (2d) is considered. If the parameters satisfy $a>0, b>0$, and $c>0$, then the following conditions are met as

$$
=\frac{d\left(1+a c+2 d-a^{2} d+a c d+d^{2}-a k-c k+a d k-c d k+k^{2}\right)}{(1+d)^{2}},
$$

and system (2a), (2b), (2c), and (2d) has no bounded chaotic solutions or hyperchaotic solutions. 
Proof. From system (2a), (2b), (2c), and (2d),

$$
\begin{aligned}
d \dot{x}+\frac{d(k-a)}{1+d} \dot{z}+\dot{u}= & (-a d-s) x+(d-m-c s) z \\
& +(-k+s) u .
\end{aligned}
$$

Under assumption (3), (4) becomes

$$
\begin{aligned}
d \dot{x} & +\frac{d(k-a)}{1+d} \dot{z}+\dot{u} \\
& =-\frac{a d+k}{1+d}\left[d x+\frac{d(k-a)}{1+d} z+u\right] .
\end{aligned}
$$

Thus, we can get the expression that

$$
\begin{aligned}
d x & +\frac{d(k-a)}{1+d} z+u \\
& =\left[d x(0)+\frac{d(k-a)}{1+d} z(0)+u(0)\right] e^{-((a d+k) /(1+d)) t} .
\end{aligned}
$$

Since $(a d+k) /(1+d)<0(a>0$ is the amount of saving; $b>0$ is the cost per investment) and at least one of the following conditions is satisfied: $x(t), z(t)$, or $u(t)$ is not bounded, then system (2a), (2b), (2c), and (2d) is not chaotic.

The proof is complete.

\section{Some Basic Properties and Bifurcation Analysis of the New System (2a), (2b), (2c), and (2d)}

3.1. Equilibria and Stability. Firstly, the invariance of system is easily demonstrated with the transformation of coordinate $(x, y, z, u) \rightarrow(-x,-y, z,-\omega)$; namely, the system has rotated symmetry around the axis $z$.

We consider the equilibrium point of system (2a), (2b), (2c), and (2d) to analyze the equilibria and let

$$
\begin{aligned}
z+(y-a) x & =0, \\
1-b y-x^{2} & =0, \\
-x-c z+u & =0, \\
-d x y-k u-m z & =0 .
\end{aligned}
$$

Combining (7a), (7b), and (7c), we obtain

$$
\begin{aligned}
& x= \pm \sqrt{1-b y}, \\
& z= \pm(a-y) \sqrt{1-b y}, \\
& u= \pm \sqrt{1-b y}(1+a c-c y) .
\end{aligned}
$$

Substituting (8) into (7d) yields

$$
\sqrt{1-b y}[(d-c k-m) y+k+a c k+a m]=0,
$$

where $f_{y}^{\prime}=0$ and

$$
\begin{aligned}
f_{y}^{\prime}= & -\frac{b}{2 \sqrt{1-b y}}[(d-c k-m) y+k+a c k+a m] \\
& +\sqrt{1-b y}[d-c k-m]=0, \\
y= & \frac{2}{3 b}-\frac{k+a c k+a m}{3(d-c k-m)} .
\end{aligned}
$$

Substituting (11) into (10) yields

$$
\begin{aligned}
& \sqrt{1-\frac{b(k+a c k+a m)}{d-c k-m}}\left(\frac{d-c k-m}{b}+k+a c k\right. \\
& +a m)=0 .
\end{aligned}
$$

Hence, we can obtain the following results:

System (2a), (2b), (2c), and (2d) have only one equilibrium point $E_{0}(0,1 / b, 0,0)$.

If $d-c k-m \neq 0$ and $\Gamma_{1}=-d+c k+m$ and $\Gamma_{2}=k+a c k+a m$, system (2a), (2b), (2c), and (2d) with $0<\Gamma_{1}<b \Gamma_{2}$ has three equilibrium points $E_{0}(0,1 / b, 0,0)$ and $E_{1,2}\left( \pm x_{0}, y_{0}, \mp z_{0}, \mp u_{0}\right)$, where

$$
\begin{aligned}
& x_{0}=\frac{\Gamma_{1}-b \Gamma_{2}}{\Gamma_{1}}, \\
& y_{0}=\frac{\Gamma_{2}}{\Gamma_{1}}, \\
& z_{0}=(a d+k) \frac{\Gamma_{1}-b \Gamma_{2}}{\Gamma_{1}^{3 / 2}}, \\
& u_{0}=(d+a c d-m) \frac{\Gamma_{1}-b \Gamma_{2}}{\Gamma_{1}^{3 / 2}} .
\end{aligned}
$$

In the next step, we research the stability situation of equilibria $E_{0}$ and $E_{1,2}$. By linearizing system (2a), (2b), (2c), and $(2 \mathrm{~d})$ at the equilibrium $\left(x_{*}, y_{*}, z_{*}, u_{*}\right)$, the Jacobian matrix is

$$
J(E)=\left(\begin{array}{cccc}
y_{*}-a & x_{*} & 1 & 0 \\
-2 x_{*} & -b & 0 & 0 \\
-1 & 0 & -c & 1 \\
-d y_{*} & -d x_{*} & -m & -k
\end{array}\right) .
$$

Obviously, the characteristic equation at the equilibrium point $\left(x_{*}, y_{*}, z_{*}, u_{*}\right)$ is

$$
\begin{aligned}
\lambda^{4} & +\left(a+b+c+k-y_{*}\right) \lambda^{3}+(1+a b+a c+b c+a k \\
& \left.+b k+c k+m+2 x_{*}^{2}-b y_{*}-c y_{*}\right) \lambda^{2}+(b+a b c \\
& +k+a b k+a c k+b c k+a m+b m+2 c x_{*}^{2}-b c y_{*} \\
& \left.+d y_{*}-b k y_{*}-c k y_{*}-m y_{*}\right) \lambda+b k+a b c k+a b m \\
& -2 d x_{*}^{2}+2 c k x_{*}^{2}+2 m x_{*}^{2}+b d y_{*}-b c k y_{*}-b m y_{*} \\
& =0 .
\end{aligned}
$$


The following symbols are introduced:

$$
\begin{aligned}
a_{1}= & a+b+c+k-y_{*}, \\
a_{2}=1 & +a b+a c+b c+a k+b k+c k+m+2 x_{*}^{2} \\
& -b y_{*}-c y_{*}, \\
a_{3}= & +a b c+k+a b k+a c k+b c k+a m+b m \\
& +2 c x_{*}^{2}-b c y_{*}+d y_{*}-b k y_{*}-c k y_{*}-m y_{*}, \\
a_{4}= & b k+a b c k+a b m-2 d x_{*}^{2}+2 c k x_{*}^{2}+2 m x_{*}^{2} \\
& +b d y_{*}-b c k y_{*}-b m y_{*} .
\end{aligned}
$$

By using the criterion initiated by Routh-Hurwitz, the real parts of all the roots are negative if and only if

$$
\begin{aligned}
a_{i} & >0, \quad(i=1,2,3,4) \\
a_{1} a_{2}-a_{3} & >0, \\
a_{1} a_{2} a_{3}-a_{3}^{2}-a_{1}^{2} a_{4} & >0 .
\end{aligned}
$$

Therefore, the equilibrium point $\left(x_{*}, y_{*}, z_{*}, u_{*}\right)$ is asymptotically stable when (17) are satisfied.

Remark 2. For the sake of simplification of the study of dynamical behavior of system (2a), (2b), (2c), and (2d) in the following subsections, the values of system parameters are fixed as $a=0.9, c=1.5, d=0.2$, and $k=0.05$.

(A) When $m=0.005$, system (2a), (2b), (2c), and (2d) has three equilibria for $E_{0}$ and $E_{1,2}$ :

$\left(A_{1}\right) E_{0}$ is asymptotically stable when $b>0.7068$.

$\left(A_{2}\right) E_{1,2}$ is unstable for $b \in R^{+}$as $a_{1} a_{2}-a_{3}<0$.

(B) When $b=0.2$, system (2a), (2b), (2c), and (2d) has three equilibria for $E_{0}$ and $E_{1,2}$ :

$\left(B_{1}\right) E_{0}$ is unstable for $m \in R^{+}$as $a_{1}<0$.

$\left(B_{2}\right) E_{1,2}$ is asymptotically stable when $m>0.5139$.

With the target of finding the effect of control parameters $b$ of the new dynamic system of four dimensions, the simulation results are obtained by numerical simulation. According to conditions $\left(A_{2}\right)$ and $\left(B_{1}\right)$, some dynamic properties of system $(2 \mathrm{a}),(2 \mathrm{~b}),(2 \mathrm{c})$, and $(2 \mathrm{~d})$ can be analyzed through bifurcation diagrams.

3.2. An Analysis of System Bifurcation (2a), (2b), (2c), and (2d). Now we calculate the first Lyapunov coefficient in relation to Hopf bifurcation by employing the projection method [25]. Let $l_{1}$ be the first Lyapunov coefficient related to Hopf bifurcation. Firstly, consider the differential equation

$$
\dot{X}=f(X, \mu),
$$

in which $x \in R^{4}$ denotes vectors representing phase variables and $\mu \in R^{6}$ denotes control parameters. Suppose $f$ is a class of $C^{\infty}$ in $R^{4} \times R^{6}$ and there is an equilibrium point in (18) $X=X_{0}$ at $\mu=\mu_{0}$. Let variable $X-X_{0}$ be $X$, and we can write

$$
F(X)=f\left(X, \mu_{0}\right)
$$

as

$$
\begin{aligned}
F(X)= & A X+\frac{1}{2} B(X, X)+\frac{1}{6} C(X, X, X) \\
& +O\left(\|X\|^{4}\right)
\end{aligned}
$$

where $A=f_{x}\left(0, \mu_{0}\right)$ and, for $i=1,2,3$,

$$
\begin{gathered}
B(X, Y)=\left.\sum_{j, k=1}^{3} \frac{\partial^{2} F_{i}(\xi)}{\partial \xi_{i} \partial \xi_{k}}\right|_{\xi=0} X_{j} Y_{k}, \\
C(X, Y, Z)=\left.\sum_{j, k, l=1}^{3} \frac{\partial^{3} F_{i}(\xi)}{\partial \xi_{j} \partial \xi_{k} \partial \xi_{l}}\right|_{\xi=0} X_{j} Y_{k} Z_{l} .
\end{gathered}
$$

The following considerations are supposed: $A$ has a pair of complex eigenvalues on the imaginary axis $\lambda_{2,3}= \pm i \omega\left(\omega_{0}>\right.$ 0 ), which are the only eigenvalues with $\operatorname{Re} \lambda=0, T^{c}$ is the generalized eigenspace of $A$ with regard to $\lambda_{2,3}$, and $p, q \in C^{3}$ are vectors such that

$$
\begin{aligned}
A q & =i \omega_{0} q, \\
A^{T} p & =-i \omega_{0} p, \\
\langle p, q\rangle & =1,
\end{aligned}
$$

in which $A^{\mathrm{T}}$ is the transposition of the matrix $A$.

Vector $y \in T^{c}$ can be showed as $y=\omega q+\bar{\omega} \bar{q}$ with $\omega=\langle p, y\rangle \in C$. The two-dimensional center manifold related to the eigenvalues $\lambda_{2,3}$ is parameterized by $\omega$ and $\bar{\omega}$, with an immersion of the form $X=H(\omega, \bar{\omega})$, where $H: C^{2} \rightarrow R^{3}$ is a Taylor expansion of the form

$$
\begin{aligned}
H(\omega, \bar{\omega})= & \omega q+\bar{\omega} \bar{q}+\sum_{2 \leq j+k \leq 3} \frac{1}{j ! k !} h_{j k} \omega^{j} \bar{\omega}^{k} \\
& +o\left(|\omega|^{4}\right)
\end{aligned}
$$

with $h_{j k} \in C^{3}$ and $h_{j k}=\bar{h}_{k j}$. Substituting (23) into (19), the following differential equation could be reached:

$$
H_{\omega} \omega^{\prime}+H_{\omega} \bar{\omega}^{\prime}=F(H(\omega, \bar{\omega})) .
$$

By solving the system of linear equations defined by the coefficients of (19), we can get the complex vectors $h_{i j}$. Given the coefficient $F$, system equation (19) can be written on the chart $\omega$ for a central manifold as

$$
\dot{\omega}=i \omega_{0} \omega+\frac{1}{2} G_{21} \omega|\omega|^{2}+O\left(|\omega|^{4}\right)
$$

where $G_{21} \in C$.

Denote the first Lyapunov coefficient as

$$
l_{1}=\frac{1}{2} R_{e} G_{21}
$$

in which $G_{21}=\left\langle p, C(q, q, \bar{q})+B\left(\bar{q}, h_{20}\right)+2 B\left(q, h_{11}\right)\right\rangle$. 
A Hopf bifurcation point $\left(X_{0}, \mu_{0}\right)$ is an equilibrium point of (18), a pair of purely imaginary eigenvalues $\pm i \omega_{0}(\omega>0)$ and another eigenvalue with nonzero real part only exists in the Jacobian matrix $A$. A two-dimensional center manifold is well defined at a Hopf point, which is invariant under the flow produced by (18) and can continue with arbitrarily high class of differentiability to nearby parameter values.

If the parameter-dependent complex eigenvalues cross the imaginary axis with nonzero derivative, then a Hopf point is called transversal. In the area of a transversal Hopf point with $l_{1} \neq 0$, the dynamic behavior of system (18) can decrease to a family of parameter-dependent continuations of the center manifold and is topologically orbital equivalent to the complex normal form

$$
\omega^{\prime}=(\eta+i \omega) \omega+l_{1} \omega|\omega|^{2}
$$

where $\omega \in C$ and $\eta, \omega$, and $l_{1}$ are real functions with arbitrarily higher order derivatives, which are continuations of $0, \omega_{0}$, and the first Lyapunov coefficient at the Hopf point [23]. When $l_{1}<0\left(l_{1}>0\right)$, we can find one family of stable (unstable) periodic orbits on this manifolded family, contracting to an equilibrium point at the Hopf point.

The remaining part of this section employs the fourdimensional Hopf bifurcation theory and uses symbolic computations to carry out the analysis of parametric variations concerning dynamical bifurcations. Because the system has only one equilibrium, the bifurcation of system (2a), (2b), $(2 \mathrm{c})$, and $(2 \mathrm{~d})$ will be our only concern, and then we get Theorem 3.

\subsubsection{Hopf Bifurcation at $E_{0}$}

Theorem 3. With system (2a), (2b), (2c), and (2d) and $a=0.9$, $c=1.5, d=0.2, k=0.05$, and $m=0.005$, the first Lyapunov coefficient at $E_{0}$ for critical value $b=b_{0}=0.7068$ is given by

$$
l_{1}=-0.1568<0 \text {. }
$$

Therefore, there exists a transversal Hopf point at $E_{0}$ of system (2a), (2b), (2c), and (2d), and thus this point is stable. Moreover, for each $b<b_{0}$, but close to $b_{0}$, there is a stable limit cycle close to the unstable equilibrium point $E_{0}$.

Proof. As to the parameters $(a, c, d, k, m)=(0.9,1.5,0.2,0.05$, $0.005)$ and $b=b_{0}=0.7068$, we have

$$
\begin{aligned}
\lambda_{1} & =-1.0351, \\
\lambda_{2} & =-0.7068, \\
\lambda_{3,4} & = \pm 0.5309 i .
\end{aligned}
$$

Under this condition, it is easy to know the transverse condition $\lambda^{\prime}\left(b=b_{0}\right)<0$. Accordingly, there exists a Hopf bifurcation at $E_{0}$. The stability of $E_{0}$ can be decided by the value of the first Lyapunov coefficient $l_{1}$ and $l_{1}$ can show the stability of $E_{0}$ and the occurred periodic orbits. Making use of the notation of the section above, the multilinear symmetrical functions could be written as

$$
\begin{aligned}
& B(x, y) \\
& \quad=\left(x_{1} y_{3}+x_{3} y_{1},-2 x_{1} y_{1}, 0,-d x_{1} y_{2}-d x_{2} y_{1}\right), \\
& C(x, y, z)=(0,0,0,0) .
\end{aligned}
$$

Moreover, the following results are obtained:

$$
\begin{aligned}
p & =(0.142675+0.581567 i, 0,-0.0353347 \\
& +0.375204 i,-0.70668), \\
q & =(0.522813+1.01926 i, 0,-0.810362 \\
& -0.247239 i,-0.561461+0.218144 i), \\
h_{11} & =(-0.370497,-3.71333,1.54211,1.94266), \\
h_{20} & =(-2.77049+1.20144 i,-0.726017 \\
& -1.92509 i, 0.494102-1.6501 i,-0.277134 \\
& -0.749036 i) .
\end{aligned}
$$

Then the following value is computed:

$$
\begin{aligned}
G_{21} & =-0.313594-2.65646 i, \\
l_{1} & =\frac{1}{2} R_{e} G_{21}=-0.1568 .
\end{aligned}
$$

Therefore, the theorem is proved. Near the unstable equilibrium point $E_{0}$, we can find a stable periodic solution for $b<b_{0}$.

\subsubsection{Hopf Bifurcation at $E_{1,2}$}

Theorem 4. With system (2a), (2b), (2c), and (2d) and $a=0.9$, $c=1.5, d=0.2, k=0.05$, and $b=0.2$, the first Lyapunov coefficient at $E_{1,2}$ for critical value $m=m_{0}=0.5139$ is given by

$$
l_{1}=-0.3045<0 \text {. }
$$

Therefore, system (2a), (2b), (2c), and (2d) has a transversal Hopf point at $E_{1,2}$, and thus this point is stable. Furthermore, for each $m<m_{0}$, but close to $m_{0}$, a stable limit cycle exists near the unstable equilibrium point $E_{1,2}$.

Proof. Because of symmetry, $E_{1}$ will be considered. Since the parameters satisfy $(a, c, d, k, b)=(0.9,1.5,0.2,0.05,0.2)$ and $m=m_{0}=0.5139$, we have

$$
\begin{aligned}
\lambda_{1} & =-0.8279, \\
\lambda_{2} & =-0.3307, \\
\lambda_{3,4} & = \pm 1.4120 i .
\end{aligned}
$$

Under this condition, it is easy to know the transverse condition $\lambda^{\prime}\left(m=m_{0}\right)<0$. Accordingly, Hopf bifurcation 
appears. There exists a Hopf bifurcation at $E_{0}$. The value of the first Lyapunov coefficient $l_{1}$ can decide the stability of $E_{0}$. It demonstrates the stability of the periodic orbits and the equilibrium point. Making use of the notation obtained from the section above, the multilinear symmetric functions could be written as

$$
\begin{aligned}
& B(x, y) \\
& \quad=\left(x_{1} y_{3}+x_{3} y_{1},-2 x_{1} y_{1}, 0,-d x_{1} y_{2}-d x_{2} y_{1}\right), \\
& C(x, y, z)=(0,0,0,0) .
\end{aligned}
$$

Then, the following results are obtained:

$$
\begin{aligned}
p= & (0.741494,-0.0915377+0.445225 i, 0.331105 \\
& -0.22946 i,-0.154015,-0.239956 i) \\
q & =(0.6928-0.277114 i,-0.208195-0.851544 i, \\
& -0.192858+0.428759 i,-0.201872 \\
& +0.0937186 i), \\
h_{11} & =(0.542254,-1.02525,-0.674678,-0.469763), \\
h_{20} & =(0.218437+0.0754352 i, 0.304126 \\
& +0.177492 i,-0.0578508 \\
& +0.0199395 i, 0.075354-0.0580204 i) .
\end{aligned}
$$

Then the following values are computed:

$$
\begin{aligned}
G_{21} & =-0.608777+1.36819 i, \\
l_{1} & =\frac{1}{2} R_{e} G_{21}=-0.3045 .
\end{aligned}
$$

Therefore, the theorem is justified. We can find a stable periodic solution close to the unstable equilibrium point $E_{1}$ for $m<m_{0}$.

\section{Four-Dimensional Estimate of Ultimate Bound and Positively Invariant Set}

Let $X=\left[x_{1}, x_{2}, x_{3}, x_{4}\right]^{\mathrm{T}}$. Define $X\left(t, t_{0}, X_{0}\right)$ as the solution to system as

$$
\dot{X}=f(X, \mu), \quad X \in R^{4},
$$

and $X\left(t, t_{0}, X_{0}\right)=X_{0}$ at the initial time $t_{0}$ and initial state $X_{0}$, which is denoted as $X(t)$ for simplicity. $\Omega \in R^{4}$ is assumed as a compact set, and the distance between the solution $X(t)$ and the set $\Omega$ by $\rho(X(t), \Omega)=\inf _{Y \in \Omega}\|X(t)-y\|$ is defined and denoted as $\Omega_{\varepsilon}=\{X \mid \rho(X, \Omega)<\varepsilon\}$.

Definition 5 (see $[26,27]$ ). Supposing a compact set $\Omega \in R^{4}$. If, for every $X_{0} \in R^{4} / \Omega, \lim _{t \rightarrow \infty} \rho(X(t), \Omega)=0$, that means, for any $\varepsilon>0$, there is $T>t_{0}$, such that, for $t>T$, $X\left(t, t_{0}, X_{0}\right) \in \Omega_{\varepsilon}$, and then the set $\Omega$ is named an ultimate bound for system (36a), (36b), (36c), and (36d); if for any $X_{0} \in \Omega$ and all $t \geq t_{0}, X\left(t, t_{0}, X_{0}\right) \subset \Omega$, then $\Omega$ is called the positively invariant set for system (38).
Since quadratic polynomial type Lyapunov functions are simple both in form and in application, we mainly probe into the bounds of chaotic systems by using conicoids. So we choose Lyapunov function as

$$
p(X)=X^{\mathrm{T}} P X+\zeta X+a_{0}
$$

in which $P=\left(a_{i j}\right)_{4 \times 4} \in R^{4 \times 4}, \zeta=\left(\zeta_{1}, \zeta_{2}, \zeta_{3}, \zeta_{4}\right) \in R^{4 \times 4}$, and $a_{0} \in R$ are parameters to determine. Deriving $p(X)$ along system (38) and deleting the terms with three or higher degrees, we get

$$
\dot{p}=X^{\mathrm{T}} Q X+\eta X+b_{0}
$$

Here $Q=\left(b_{i j}\right)_{4 \times 4} \in R^{4 \times 4}, \eta=\left(\eta_{1}, \eta_{2}, \eta_{3}, \eta_{4}\right) \in R^{4 \times 4}$, and $b_{0} \in R$.

If $P$ is positive definite and $Q$ is negative definite, then $\dot{p}=0$ must be a bounded sphere in $R^{4}$; and now that chaotic systems are bounded, $p(X)$ reaches the maximum or minimum values under the solution set of (18), whereas it is necessary that $\dot{p}=0$. So, in order to assess the ultimate bounds of system equation (18), the following optimization problem should only be solved:

$$
\begin{array}{ll}
\text { Maximum (or minimum) } & p(X) \\
& =X^{\mathrm{T}} P X+\zeta X+a_{0} \\
& X^{\mathrm{T}} \mathrm{Q} X+\eta X+b_{0}=0 .
\end{array}
$$

If the above discussed conditions are satisfied, then the above optimization problem has a solution supposing $p_{\min } \leq$ $p(X) \leq p_{\max }$, and then set $\Omega=\left\{X \in R^{4} \mid p_{\min } \leq p(X) \leq\right.$ $\left.P_{\max }\right\}$ would be our desired ultimate bounds.

Making use of the above method, we talk about the ultimate bound and positively invariant set for system (2a), (2b), (2c), and (2d) with $d=0$.

Theorem 6. Denote $\Omega=\left\{(x, y, z, u) \mid m x^{2}+m(y-h)^{2}+m z^{2}+\right.$ $\left.u^{2} \leq R^{2}\right\}$, where

$$
R^{2}= \begin{cases}\frac{m}{4 a(b-a)}, & b \geq 2 a \\ \frac{m}{4 k(b-k)}, & b \geq 2 k \\ \frac{m}{4 c(b-c)}, & b \geq 2 c \\ \frac{m}{b^{2}}, & b<2 a, b<2 k, b<2 c .\end{cases}
$$

If parameters of the system satisfy $m>0, a>0, b>0$, $c>0$, and $k>0$, then system (38) would have an ultimate bound and positively invariant set: hyperellipsoid $\Omega$.

Proof. First the following generalized positive definite and radially unbounded Lyapunov function are defined as

$$
V(x, y, z, u)=m x^{2}+m y^{2}+m z^{2}+u^{2},
$$




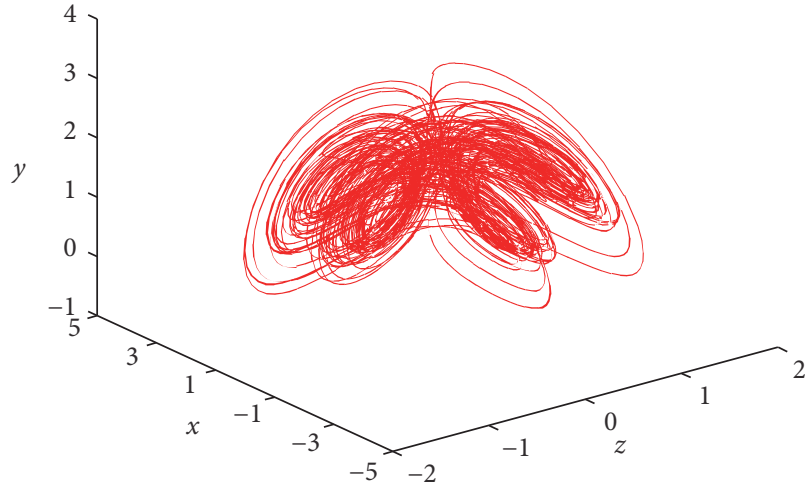

(a)

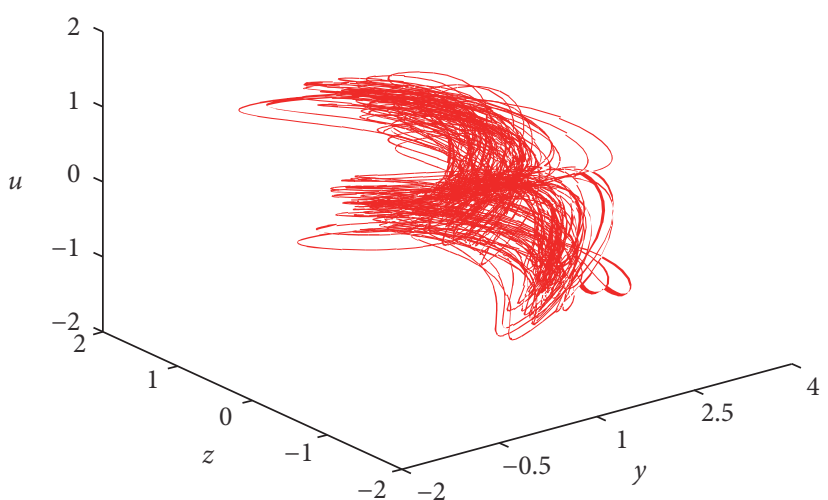

(b)

FIGURE 1: Hyperchaotic attractor for the four-dimensional financial system (2a), (2b), (2c), and (2d) with parameter values $a=0.9, b=0.2$, $c=1.5, d=0.2, k=0.05$, and $m=0.005$ and the initial condition $(0,1,-0.5,0)$. (a) The phase portrait in $(x, y, z)$ space and $u=0$; (b) the phase portrait in $(y, z, u)$ space and $x=0$.

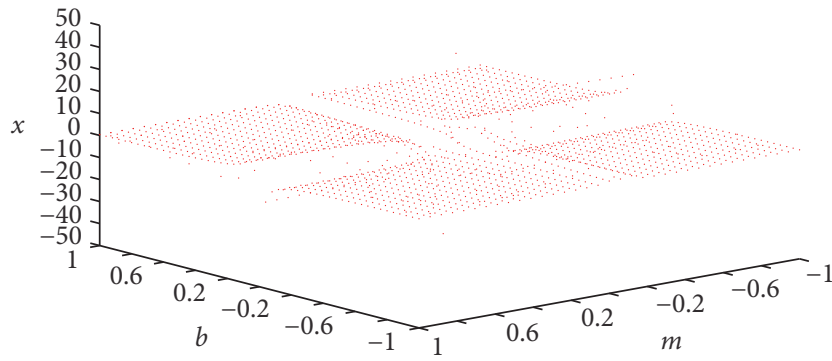

FIGURE 2: The bifurcation set of system (2a), (2b), (2c), and (2d) with parameter values $a=0.9, c=1.5, d=0.2$, and $k=0.05$, and $b \in[-1,1]$ and $m \in[-1,1]$.

where $m>0$ and $h \in R$. Calculating the derivative of $V(x, y$, $z, u)$ along with a track of (2a), (2b), (2c), and (2d), we have

$$
\begin{aligned}
\frac{1}{2} \frac{V(x, y, z, u)}{d t}= & -a m x^{2}-m b\left(y-\frac{1}{2 b}\right)^{2}-c m z \\
& -k u^{2}+\frac{m}{4 b} .
\end{aligned}
$$

It is easy to see that, for $m>0, a>0, b>0, c>0$, and $k>$ $0, V(x, y, z, u)$ is positive definite and the quadratic principal part of $V(x, y, z, u) / d t$ is negative definite. $V(x, y, z, u)=0$, which indicates the surface

$$
\begin{aligned}
\Gamma & =\left\{(x, y, z, u) \mid a m x^{2}+m b\left(y-\frac{1}{2 b}\right)^{2}+c m z\right. \\
& \left.+k u^{2}=\frac{m}{4 b}\right\}
\end{aligned}
$$

is an ellipsoid in the space of four dimensions for certain $\sigma$, $k, b, d$, and $k$. Outside of $\Gamma, V(x, y, z, u) / d t<0$, and, inside of $\Gamma, V(x, y, z, u) / d t>0$. Thus, system (2a), (2b), (2c), and (2d) would reach its ultimate bound on $\Gamma$.

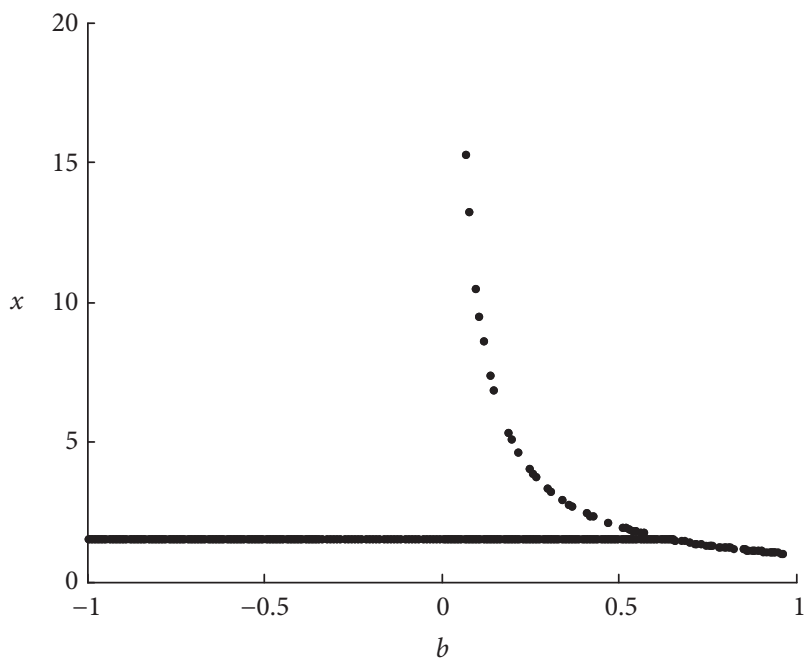

FIGURE 3: The bifurcation curve of system (2a), (2b), (2c), and (2d) with parameter values $a=0.9, c=1.5, d=0.2, k=0.05$, and $m=0.005$, and the bifurcation point occurs when $b=0.68$.

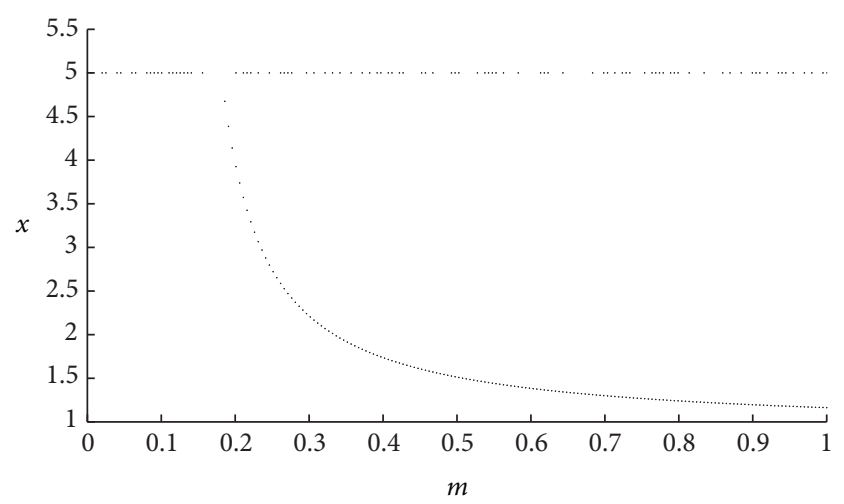

FIgURE 4: The bifurcation curves of system (2a), (2b), (2c), and (2d) with parameter values $a=0.9, c=1.5, d=0.2, k=0.05$, and $b=0.2$, and the bifurcation point occurs when $m=0.21$. 


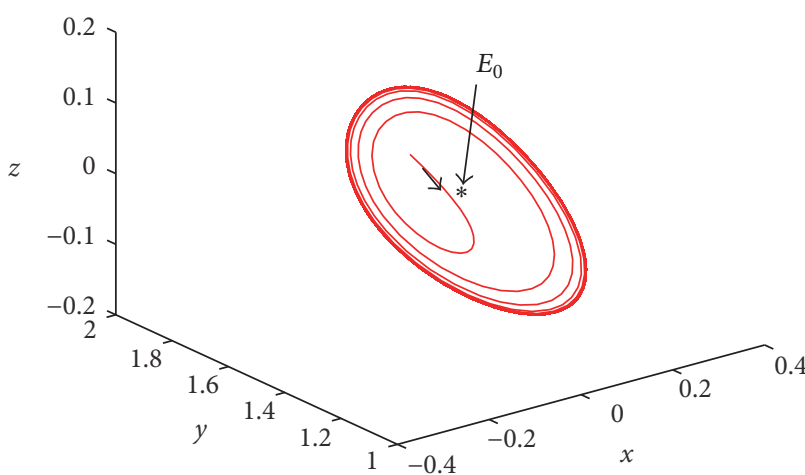

(a)

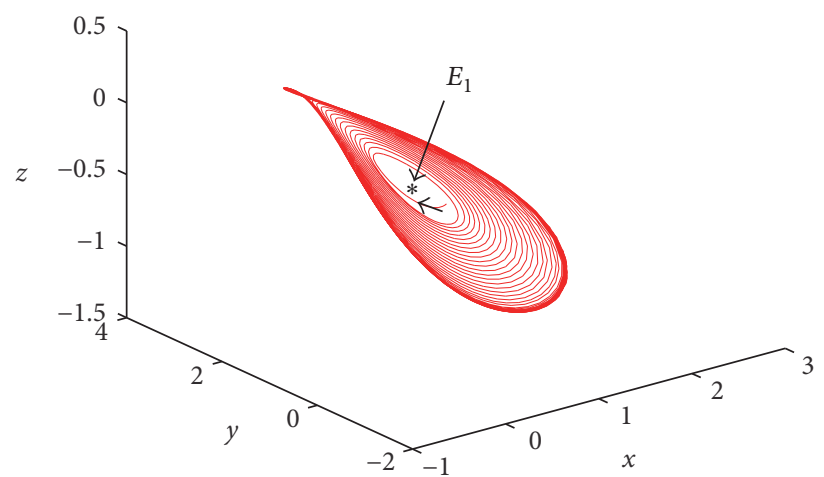

(b)

FIGURE 5: The stable periodic solution of system (2a), (2b), (2c), and (2d) with $(a, c, d, k)=(0.9,1.5,0.2,0.05)$ and $(\mathrm{a}) b=0.68$; (b) $m=0.49$.

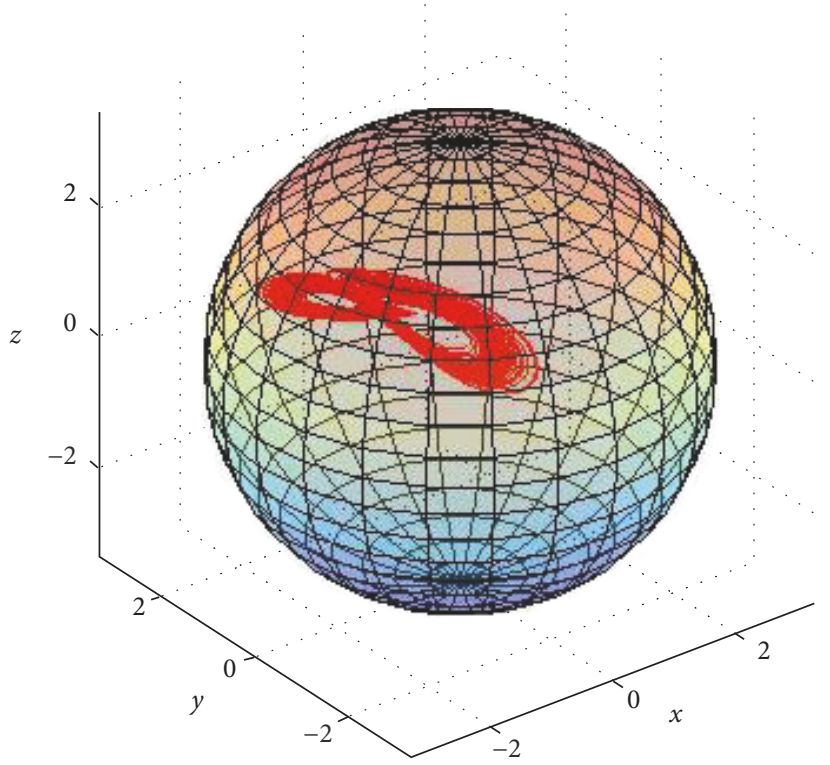

(a)

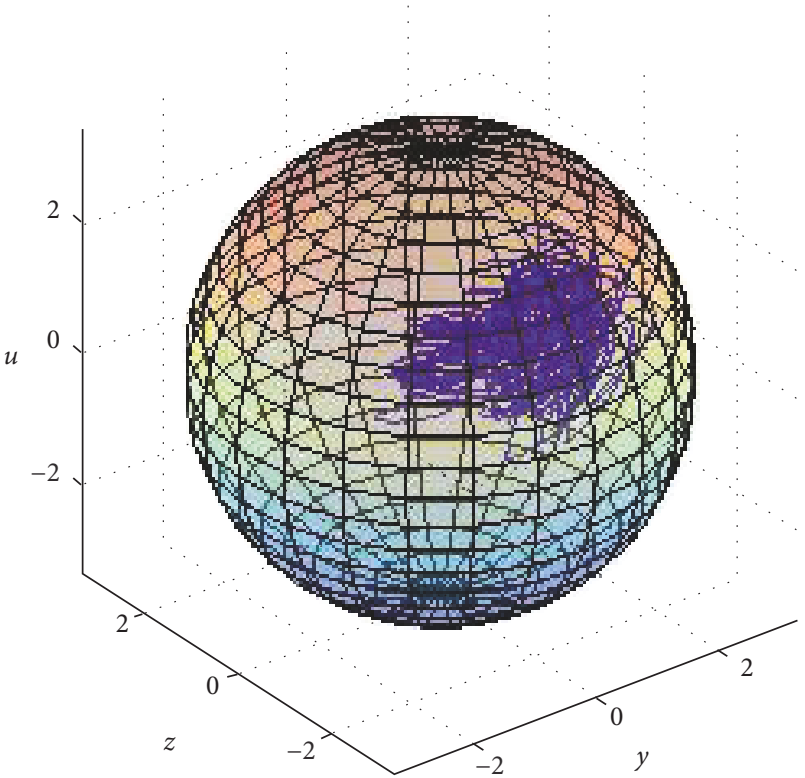

(b)

Figure 6: The projections of the bound estimated for system (2a), (2b), (2c), and (2d) with $a=0.9, b=0.3, c=1.5, d=0, k=0.05$, and $m=0.005$, (a) the phase portrait in $(x, y, z)$ space and $u=0$, and (b) the phase portrait in $(y, z, u)$ space and $x=0$.

Next we can obtain the maximum of $V$ on $\Gamma$ by expressions (2a), (2b), (2c), and (2d) according to employing the Lagrange multiplier method. Define

$$
\begin{aligned}
& F=m x^{2}+m(y-h)^{2}+m z^{2}+u^{2} \\
& +\tau\left[a m x^{2}+m b\left(y-\frac{1}{2 b}\right)^{2}+c m z+k u^{2}-\frac{m}{4 b}\right], \\
& \frac{1}{2} F_{x}^{\prime}=m(1+a \tau) x=0, \\
& \frac{1}{2} F_{y}^{\prime}=m\left[y+\tau\left(b y-\frac{1}{2}\right)\right]=0,
\end{aligned}
$$

$$
\begin{aligned}
& \frac{1}{2} F_{z}^{\prime}=m(1+c \tau) z=0 \\
& \frac{1}{2} F_{u}^{\prime}=(1+k \tau) u=0 \\
& \frac{1}{2} F_{\tau}^{\prime}=a m x^{2}+m b\left(y-\frac{1}{2 b}\right)^{2}+c m z+k u^{2}-\frac{m}{4 b} \\
& \quad=0 .
\end{aligned}
$$

(1) If $\tau \neq-1 / a, \tau \neq-1 / c$, and $\tau \neq-1 / k$, we have $(x, y$, $z, u)=(0,0,0,0)$ or $(0,1 / b, 0,0)$ and

$$
V(x, y, z, u)_{\max }=\frac{m}{b^{2}} .
$$



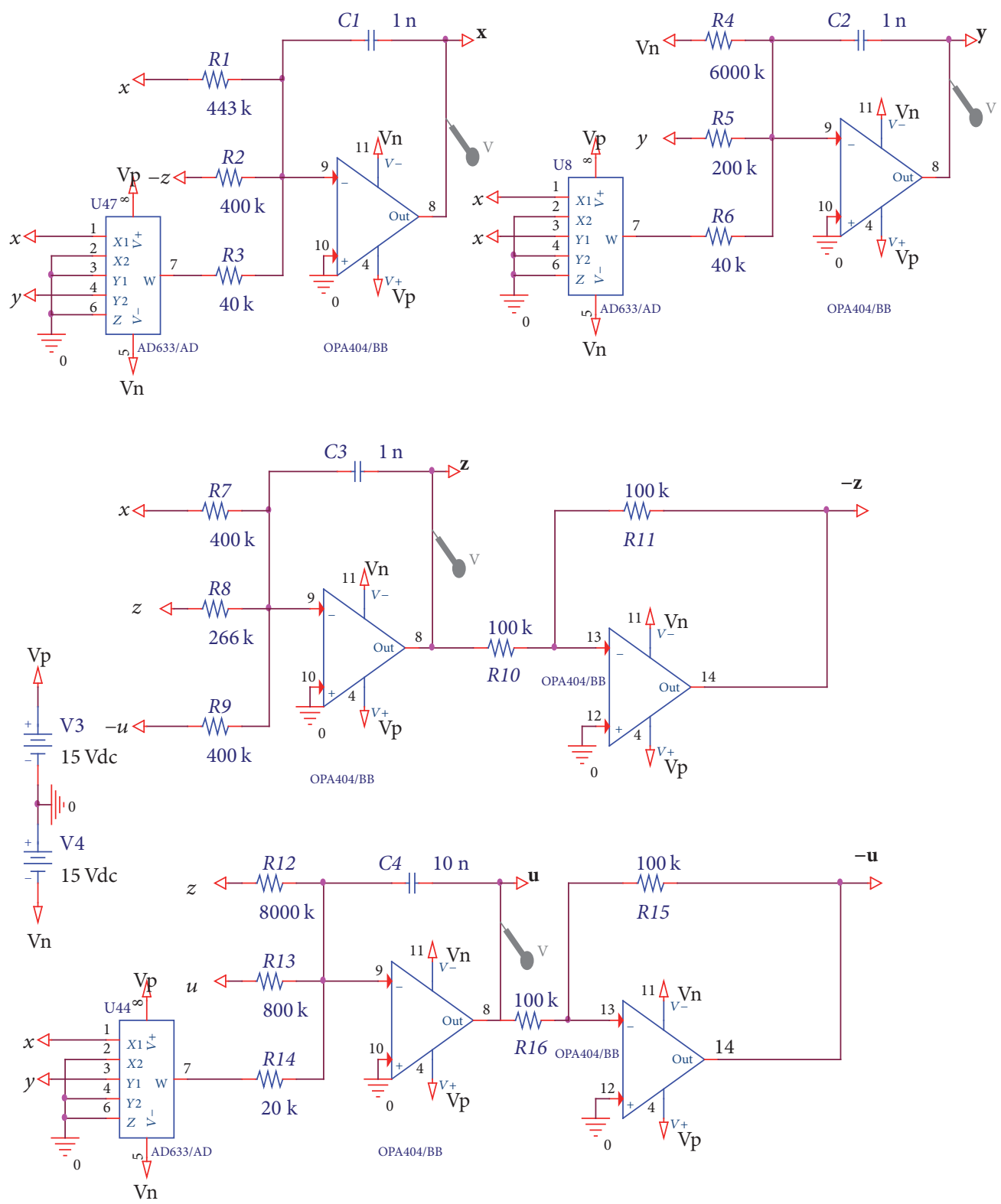

FIgURE 7: The electronic circuit schematic of the 4D financial system (2a), (2b), (2c), and (2d).

(2) If $\tau=-1 / a, \tau=-1 / c, \tau \neq-1 / k$, and $b \geq 2 a$, we have $(x, y, z, u)=\left( \pm(1 / 2) \sqrt{(b-2 a) / a(a-b)^{2}}, 1 / 2(b-a)\right.$, $0,0)$ and

$$
V(x, y, z, u)_{\max }=\frac{m}{4 a(b-a)} .
$$

(3) If $\tau=-1 / c, \tau=-1 / a, \tau \neq-1 / k$, and $b \geq 2 c$, we have $(x, y, z, u)=(0,1 / 2(b-c), \pm 1 / 2(c-b) \sqrt{(b-2 c) / c}, 0)$ and

$$
V(x, y, z, u)_{\max }=\frac{m}{4 a(b-a)} .
$$

(4) If $\tau=-1 / k, \tau \neq-1 / a, \tau \neq-1 / c$, and $b \geq 2 a$, we have $(x, y, z, u)=(0,1 / 2(b-k), 0, \pm 1 / 2(k-$ b) $\sqrt{m(b-2 k) / k})$ and

$$
V(x, y, z, u)_{\max }=\frac{m}{4 k(b-k)} .
$$

(5) If $\tau=-1 / a, a=k, k \neq c$, and $b \geq 2 a$, we have $(y, u)=$ $(1 / 2(b-a), 0), x^{2}+z^{2}=(b-2 a) / 4 a(a-b)^{2}$, and

$$
V(x, y, z, u)_{\max }=\frac{m}{4 a(b-a)} .
$$



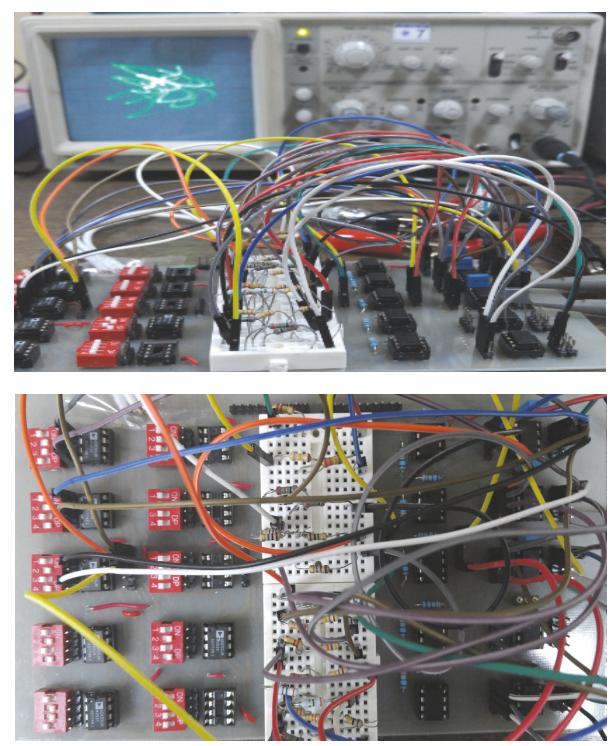

FIgURE 8: The experimental circuit of the hyperchaotic circuit.

(6) If $\tau=-1 / a, a=c, k \neq c$, and $b \geq 2 a$, we have $(y, u)=$ $(1 / 2(b-a), 0), m x^{2}+u^{2}=m(b-2 a) / 4 a(a-b)^{2}$, and

$$
V(x, y, z, u)_{\max }=\frac{m}{4 a(b-a)} .
$$

(7) If $\tau=-1 / k, a=k=q, k \neq a$, and $b \geq 2 k$, we have $y=$ $1 / 2(b-k), x^{2}+z^{2}=(b-2 a) / 4 a(a-b)^{2}$, and

$$
V(x, y, z, u)_{\max }=\frac{m}{4 k(b-k)} \text {. }
$$

(8) If $\tau=-1 / k, a=c, k \neq c$, and $b \geq 2 a$, we have $(y, u)=$ $(1 / 2(b-a), 0), u^{2}+m x^{2}+m z^{2}=m(b-2 k) / 4 k(b-k)^{2}$, and

$$
V(x, y, z, u)_{\max }=\frac{m}{4 k(b-k)} .
$$

Figure 6 performs the corresponding ultimate bound and positively invariant set $\Omega$ with $u=0$ or $x=0(a=0.9, b=0.2$, $c=1.5, d=0, k=0.05$, and $m=0.005)$. We see that the estimated bounds in Theorem 6 are feasible.

\section{Numerical Simulation}

In the previous twenty years, different tools were developed to tap the periodic responses of nonlinear dynamical systems. The periodic responses can be spotted by several traditional criteria such as bifurcation diagrams and phase portraits. In this section, we use these techniques to illustrate the existence of the periodic motions for the financial system. Based on the four-dimensional nonlinear financial system (2a), (2b), (2c), and (2d), numerical simulations have been conducted in this paper. Using MATLAB software, a numerical approach is utilized to show the nonlinear dynamic behavior in the financial system.
The red in Figure 1 shows the hyperchaotic attractors for the four-dimensional financial system (2a), (2b), (2c), and (2d) with parameter values $a=0.9, b=0.2, c=1.5$, $d=0.2, k=0.05$, and $m=0.005$ and the initial condition $(0,1,-0.5,0)$. Figure $1(\mathrm{a})$ is the phase portrait in $(x, y, z)$ space and $u=0$, and Figure $1(\mathrm{~b})$ is the phase portrait in $(y, z, u)$ space and $x=0$. It shows that the corresponding Lyapunov exponents are $L_{1}=0.03003, L_{2}=0.01448, L_{3}=$ -0.0003 , and $L_{4}=-1.2318$, and the system has complex dynamics. Figure 2 implies the bifurcation set of system (2a), (2b), (2c), and (2d) when the values of parameters are set as $a=0.9, c=1.5, d=0.2, k=0.05, b \in[-1,1]$, and $m \in$ $[-1,1]$, and the bifurcation set divides the space into three regions with three solutions above the bifurcation set, one solution in the bifurcation set, and none under the set, which is consistent with the theoretical results. Figure 3 illustrates the bifurcation curve when $a=0.9, c=1.5, d=0.2, k=0.05$, and $m=0.005$, and the point of Hopf bifurcation is $b=0.68$.

The bifurcation curve in Figure 4 shows the bifurcation with $a=0.9, c=1.5, d=0.2, k=0.05, b=0.2$, and altering system parameter $m$, and the point of Hopf bifurcation is $m=0.49$. Figure 5(a) shows that there is a stable periodic solution near the unstable equilibrium point $E_{0}$ for $(a, c, d, k)=(0.9,1.5,0.2,0.05)$ and $b=0.68$. There is a stable periodic solution near the unstable equilibrium point $E_{1}$ for $m=0.21$ shown in Figure 5(b). Figure 6 shows the corresponding ultimate bound and positively invariant set $\Omega$ with $u=0$ or $x=0(a=0.9, b=0.3, c=1.5, d=0, k=0.05$, and $m=0.005)$. Figure $6(\mathrm{a})$ is the phase portrait in $(x, y, z)$ space and $u=0$. Figure $6(\mathrm{~b})$ is the phase portrait in $(y, z, u)$ space and $x=0$. The trajectories of system (2a), (2b), (2c), and $(2 \mathrm{~d})$ are all in the domain of the estimated bound.

\section{Physical Realization for the 4D Financial System (2a), (2b), (2c), and (2d)}

In this section, the $4 \mathrm{D}$ hyperchaotic financial system will be applied for its application using the electronic circuit which has been designed on the oscilloscope. The hyperchaotic system is studied by using an electronic circuit design. The numerical simulation and the oscilloscope outputs were obtained for the similar shaped phase portraits [28-30].

The electronic circuit of the 4D financial system was designed in OrCAD-PSpice. Figure 7 shows the electronic circuit schematic of the system. Experimental electronic circuit of the $4 \mathrm{D}$ financial system was implemented for parameters $a=0.9, c=1.5, d=0.2, k=0.05$, and $m=0.005$ and initial conditions $(0,1,-0.5,0) . R 1=443 \mathrm{Kohm}, R 2=R 7$ $=R 9=400 \mathrm{Kohm}, R 3=R 6=40 \mathrm{Kohm}, R 4=6000 \mathrm{Kohm}$, $R 5=200 \mathrm{Kohm}, R 8=266 \mathrm{Kohm}, R 10=R 11=R 15=R 16$ $=100 \mathrm{Kohm}, R 12=8000 \mathrm{Kohm}, R 13=800 \mathrm{Kohm}, R 14=$ $20 \mathrm{Kohm}, C 1=C 2=C 3=1 \mathrm{nF}, C 4=10 \mathrm{nF}, V n=-15 \mathrm{~V}$, and $V p$ $=15 \mathrm{~V}$ were chosen. Real-time application of the $4 \mathrm{D}$ financial system was realized with electronic components on electronic card in Figure 8. The OrCAD-PSpice simulation outputs and oscilloscope outputs of 4D financial system, for parameters $a=0.9, c=1.5, d=0.2, k=0.05$, and $m=0.005$, are seen in Figures 9 and 10 . 

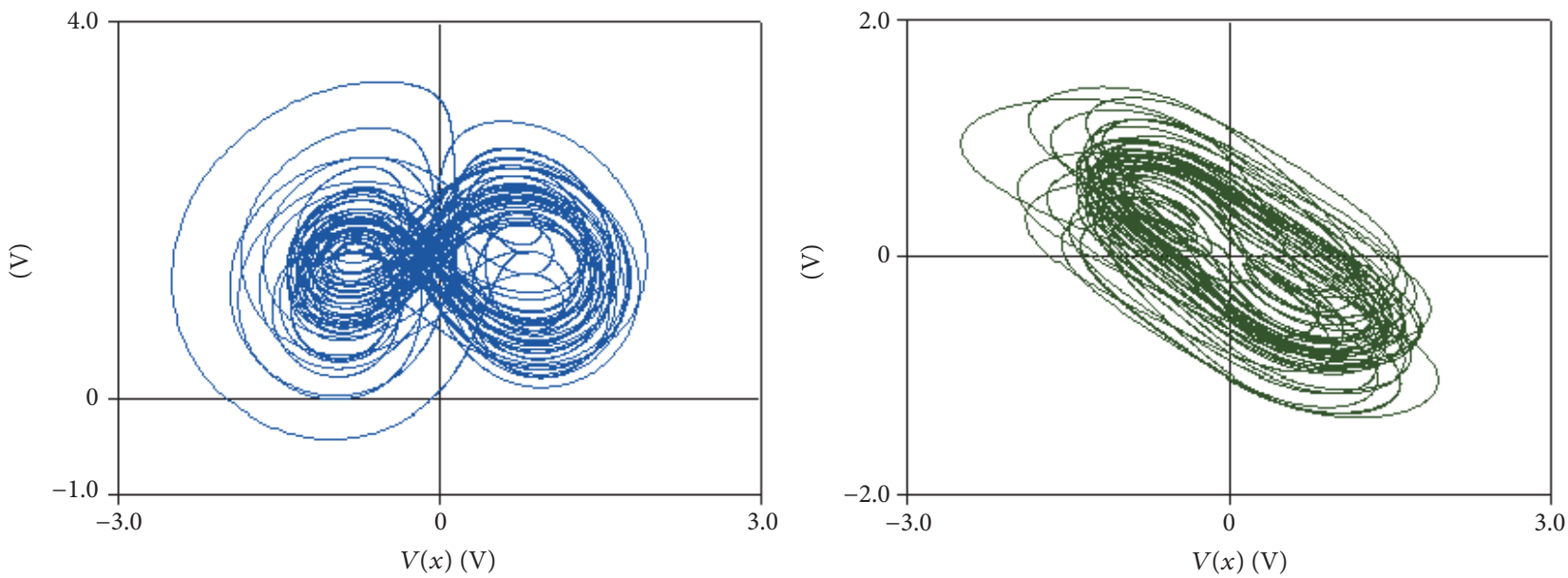

$\square V(y)$

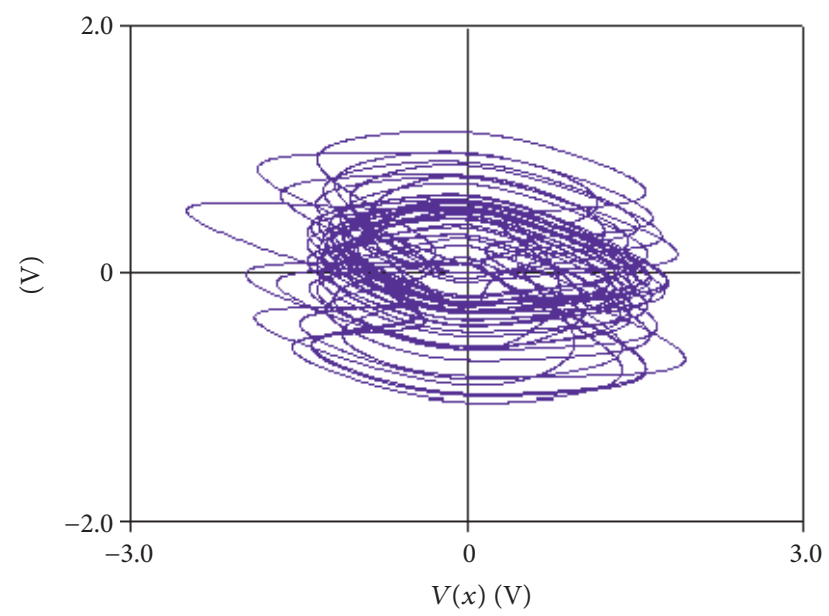

$\square V(z)$

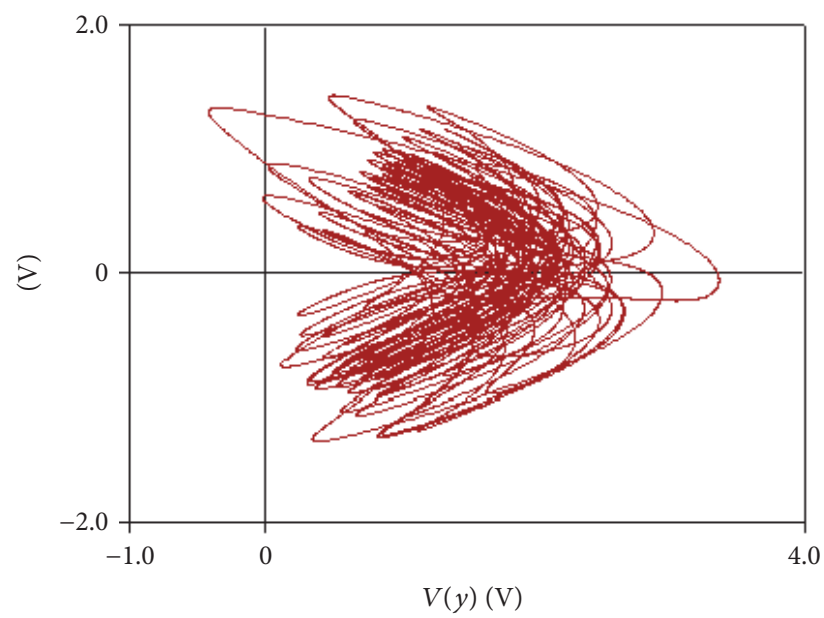

$\square V(u)$

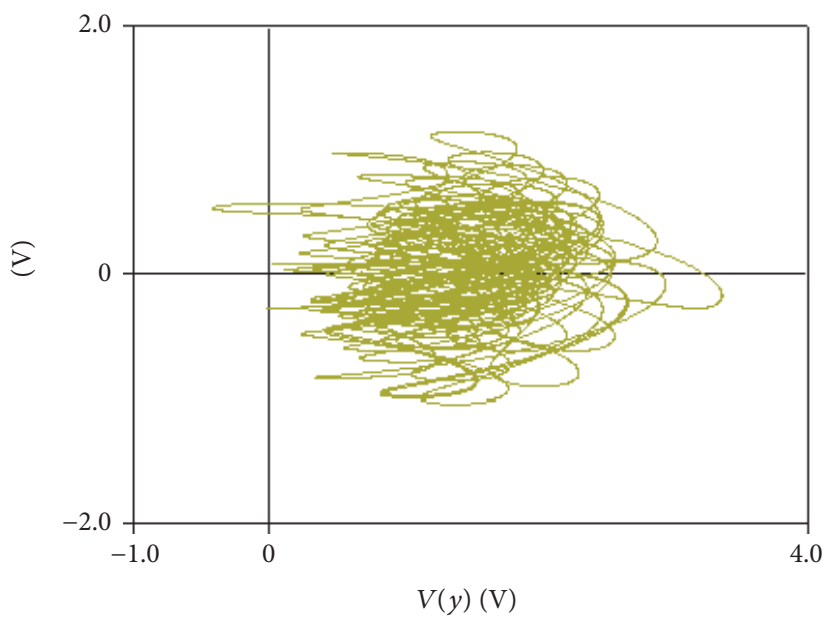

$\square V(z)$

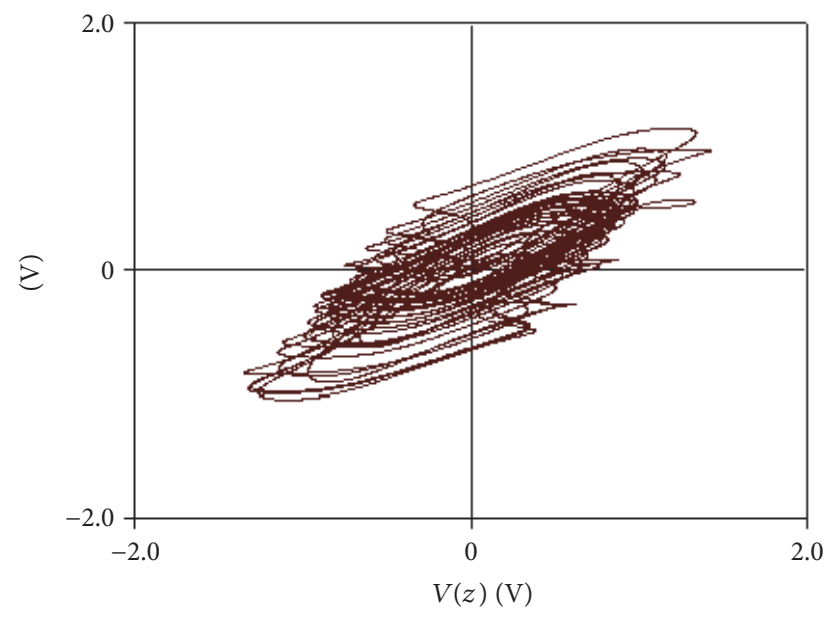

$\square V(u)$

Figure 9: The phase portraits of 4D financial system (2a), (2b), (2c), and (2d) with $a=0.9, b=0.2, c=1.5, d=0.2, k=0.05$, and $m=0.005$ in ORCAD-PSpice. 

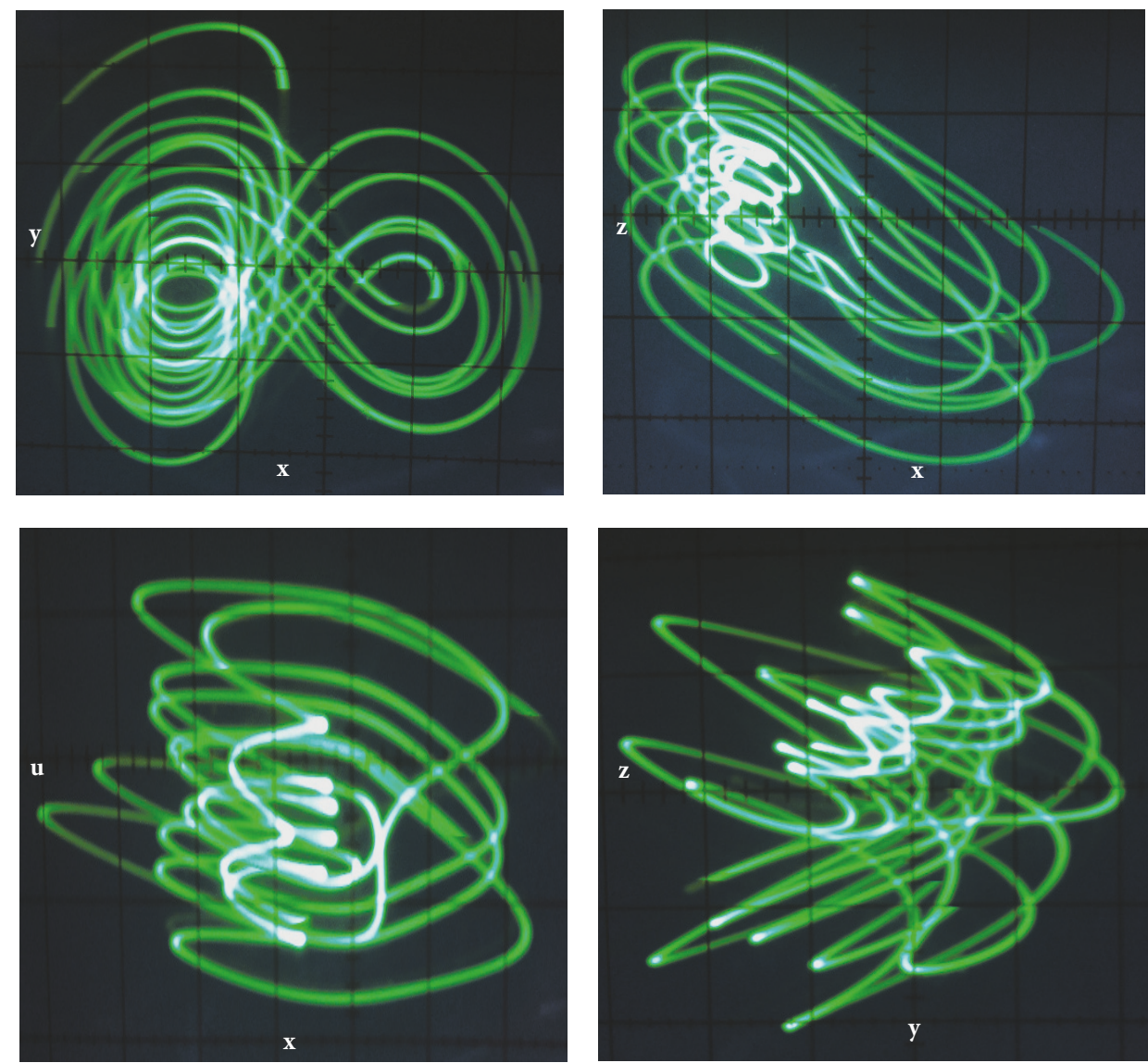

Figure 10: The phase portraits of 4D financial system (2a), (2b), (2c), and (2d) with $a=0.9, b=0.2, c=1.5, d=0.2, k=0.05$, and $m=0.005$ on the oscilloscope.

\section{Conclusion}

In our paper, the proposed financial system, which is generated from three-dimensional classical financial system, greatly expands the list of hyperchaotic financial attractors. The sufficient conditions for nonexistence of chaotic and hyperchaotic behaviors are derived theoretically, and the solutions of equilibria are obtained. For each equilibrium, its stability and existence of Hopf bifurcation are validated. Besides, based on its corresponding first Lyapunov coefficient, the analytic proof of the existence of periodic solutions is exhibited. Then the research has got the ultimate bound and positively invariant set for the proposed hyperchaotic financial system. The direction and stability of the bifurcating periodic solutions can be determined, and some numerical solutions are obtained to verify the theoretical results. In addition, the hyperchaotic financial system is extended to an electronic circuit implementation for real-time application.

We can understand the functions of financial policies and reveal the true geometrical structure of the attractors by the results of this paper. Since the global dynamics and geometrical structure of this system are not presented completely, more detailed theoretical simulation and investigations are expected in the forthcoming study.

\section{Conflicts of Interest}

The authors declare that they have no conflicts of interest.

\section{Acknowledgments}

The authors gratefully acknowledge the support of National Natural Science Foundation of China (NNSFC) through Grant nos. 11290152, 11427801, and 11401543, the Funding Project for Academic Human Resources Development in Institutions of Higher Learning under the Jurisdiction of Beijing Municipality (PHRIHLB), Beijing Postdoctoral Research Foundation (no. 2015ZZ17), the China Postdoctoral Science Foundation funded project (no. 2014M560028 and no. 2015T80029), the Government of Chaoyang District Postdoctoral Research Foundation (no. 2015ZZ-7), and Sakarya University Scientific Research Projects Unit under Grants 2016-09-00-008 and 2016-50-01-026.

\section{References}

[1] J. Alvarez-Ramirez and C. Ibarra-Valdez, "Modeling stock market dynamics based on conservation principles," Physica A: Statistical Mechanics and Its Applications, vol. 301, no. 1-4, pp. 493-511, 2001. 
[2] Q. Gao and J. Ma, "Chaos and Hopf bifurcation of a finance system," Nonlinear Dynamics, vol. 58, no. 1-2, pp. 209-216, 2009.

[3] J. H. Ma and Y. S. Chen, "Study for the bifurcation topological structure and the global complicated character of a kind of nonlinear finance system. I," Applied Mathematics and Mechanics, vol. 22, no. 11, pp. 1240-1251, 2001.

[4] J. H. Ma, Y. Q. Cui, and L. X. Liu, "Hopf bifurcation and chaos of financial system on condition of specific combination of parameters," Journal of Systems Science and Complexity, vol. 21, no. 2, pp. 250-259, 2008.

[5] C. Ma and X. Wang, "Hopf bifurcation and topological horseshoe of a novel finance chaotic system," Communications in Nonlinear Science and Numerical Simulation, vol. 17, no. 2, pp. 721-730, 2012.

[6] X.-D. Zhang, X.-D. Liu, Y. Zheng, and C. Liu, "Chaotic dynamic behavior analysis and control for a financial risk system," Chinese Physics B, vol. 22, no. 3, Article ID 030509, 2013.

[7] J. A. Holyst and K. Urbanowicz, "Chaos control in economical model by time-delayed feedback method," Physica A: Statistical Mechanics and its Applications, vol. 287, no. 3-4, pp. 587-598, 2000.

[8] K. Pyragas, "Continuous control of chaos by self-controlling feedback," Physics Letters A, vol. 170, no. 6, pp. 421-428, 1992.

[9] W.-C. Chen, "Dynamics and control of a financial system with time-delayed feedbacks," Chaos, Solitons and Fractals, vol. 37, no. 4, pp. 1198-1207, 2008.

[10] Y. Ding and J. Cao, "Bifurcation analysis and chaos switchover phenomenon in a nonlinear financial system with delay feedback," International Journal of Bifurcation and Chaos, vol. 25, no. 12, pp. 155-165, 2015.

[11] J. Yang, E. Zhang, and M. Liu, "Bifurcation analysis and chaos control in a modified finance system with delayed feedback," International Journal of Bifurcation and Chaos, vol. 26, no. 6, Article ID 1650105, 2016.

[12] J. Yoshida, "Improved criteria for hyperbolic-elliptic motion in the general three-body problem," Publications of the Astronomical Society of Japan, vol. 24, pp. 391-408, 1972.

[13] G. Seifert, "Positively invariant closed sets for systems of delay differential equations," Journal of Differential Equations, vol. 22, no. 2, pp. 292-304, 1976.

[14] B. Kalitine, "The stability of compact positively invariant sets of dynamic systems," RAIRO Automatique, vol. 16, no. 3, pp. 275286, 1982.

[15] J. Fu, L. Wang, and M. Chen, "Invariant set based sliding mode control for near-space vehicles with attitude constraints," Proceedings of the Institution of Mechanical Engineers Part G: Journal of Aerospace Engineering, vol. 230, no. 5, pp. 793-804, 2015.

[16] A. Weiss, C. Petersen, M. Baldwin, R. S. Erwin, and I. Kolmanovsky, "Safe positively invariant sets for spacecraft obstacle avoidance," Journal of Guidance, Control, and Dynamics, vol. 38, no. 4, pp. 720-732, 2015.

[17] S. V. Rakovic and R. H. Gielen, "Positively invariant families of sets for interconnected and time-delay discrete-time systems," SIAM Journal on Control and Optimization, vol. 52, no. 4, pp. 2261-2283, 2014.

[18] A. Algaba, F. F. Sanchez, M. Merino, and A. J. Rodriguez-Luis, "Comment on 'estimating the ultimate bound and positively invariant set for a synchronous motor and its application in chaos synchronization," Chaos Solitons and Fractals, vol. 44, pp. 137-144, 2011.
[19] F. Zhang, Y. Shu, H. Yang, and X. Li, "Estimating the ultimate bound and positively invariant set for a synchronous motor and its application in chaos synchronization," Chaos, Solitons and Fractals, vol. 44, no. 1-3, pp. 137-144, 2011.

[20] J. Wang, Q. Zhang, Z. Chen, and H. Li, "Local bifurcation analysis and ultimate bound of a novel 4D hyper-chaotic system," Nonlinear Dynamics, vol. 78, no. 4, pp. 2517-2531, 2014.

[21] Z. Wei and W. Zhang, "Hidden hyperchaotic attractors in a modified Lorenz-Stenflo system with only one stable equilibrium," International Journal of Bifurcation and Chaos in Applied Sciences and Engineering, vol. 24, no. 10, Article ID 1450127, 14 pages, 2014.

[22] C. Du, Y. Liu, and W. Huang, "A class of three-dimensional quadratic systems with ten limit cycles," International Journal of Bifurcation and Chaos, vol. 26, no. 9, Article ID 1650149, 2016.

[23] M. Prakash and P. Balasubramaniam, "Stability and Hopf bifurcation analysis of novel hyperchaotic system with delayed feedback control," Complexity, vol. 21, no. 6, pp. 180-193, 2016.

[24] H. Yu, G. Cai, and Y. Li, "Dynamic analysis and control of a new hyperchaotic finance system," Nonlinear Dynamics, vol. 67, no. 3, pp. 2171-2182, 2012.

[25] J. Sotomayor, L. F. Mello, and D. C. Braga, "Bifurcation analysis of the Watt governor system," Computational \& Applied Mathematics, vol. 26, no. 1, pp. 19-44, 2007.

[26] D. Li, X. Wu, and J.-A. Lu, "Estimating the ultimate bound and positively invariant set for the hyperchaotic Lorenz-Haken system," Chaos, Solitons \& Fractals, vol. 39, no. 3, pp. 1290-1296, 2009.

[27] Z. Wei, P. Yu, W. Zhang, and M. Yao, "Study of hidden attractors, multiple limit cycles from Hopf bifurcation and boundedness of motion in the generalized hyperchaotic Rabinovich system," Nonlinear Dynamics, vol. 82, no. 1-2, pp. 131-141, 2015.

[28] A. Akgul, I. Moroz, I. Pehlivan, and S. Vaidyanathan, "A new four-scroll chaotic attractor and its engineering applications," Optik, vol. 127, no. 13, pp. 5491-5499, 2016.

[29] A. Akgul, H. Calgan, I. Koyuncu, I. Pehlivan, and A. Istanbullu, "Chaos-based engineering applications with a 3D chaotic system without equilibrium points," Nonlinear Dynamics, vol. 84, no. 2, pp. 481-495, 2016.

[30] A. Akgul and I. Pehlivan, "A new three-dimensional chaotic system without equilibrium points, its dynamical analyses and electronic circuit application," Tehnicki Vjesnik, vol. 23, no. 1, pp. 209-214, 2016. 


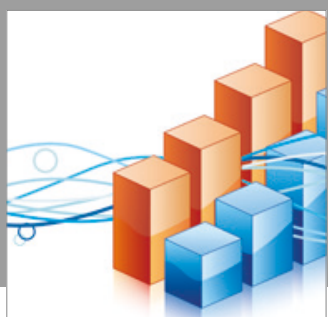

Advances in

Operations Research

vatersals

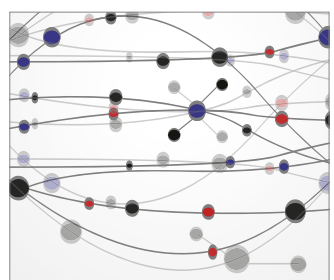

\section{The Scientific} World Journal
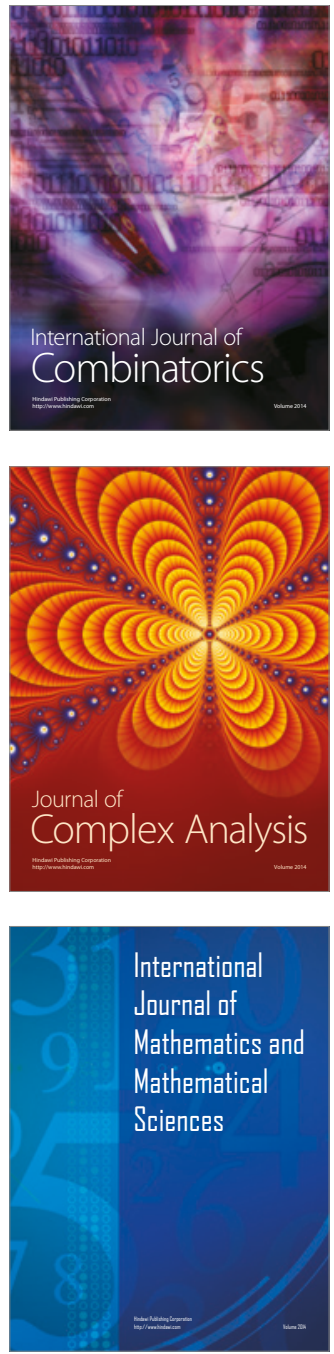
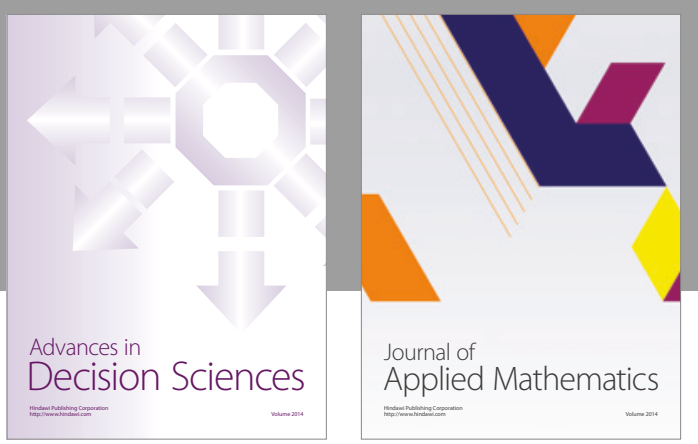

Algebra

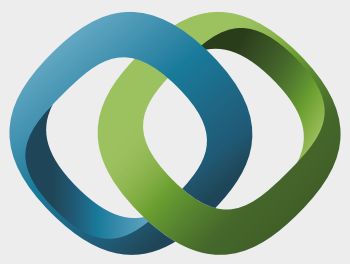

\section{Hindawi}

Submit your manuscripts at

https://www.hindawi.com
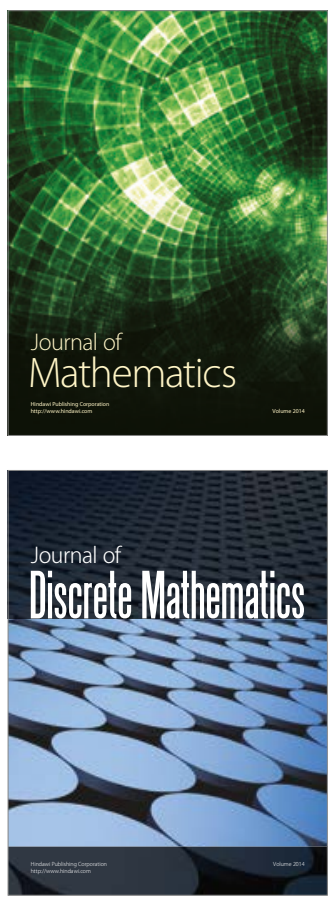

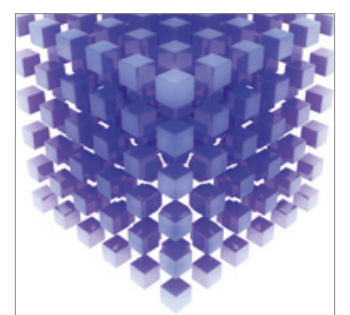

Mathematical Problems in Engineering
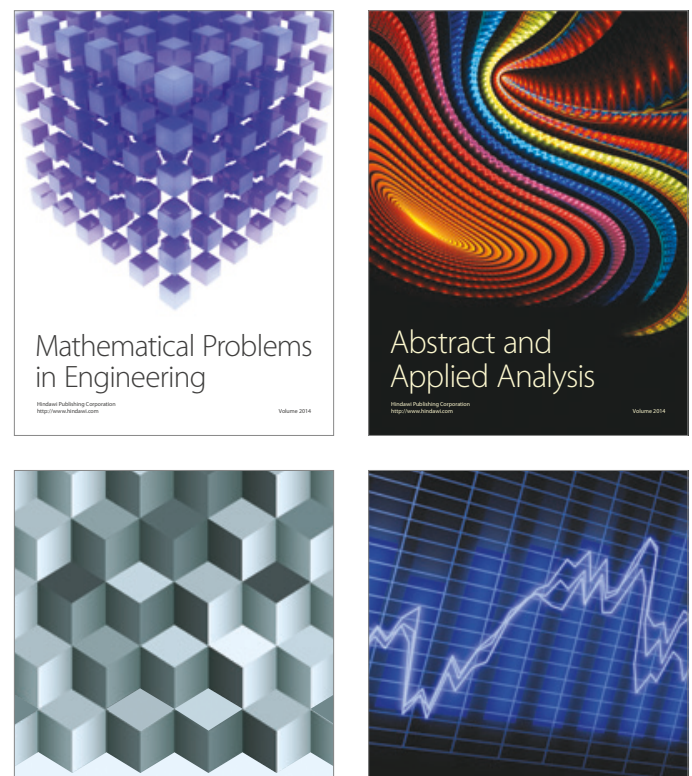

Journal of

Function Spaces

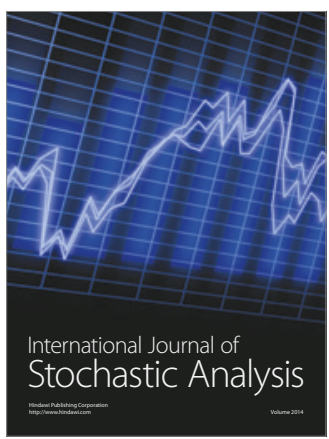

Probability and Statistics
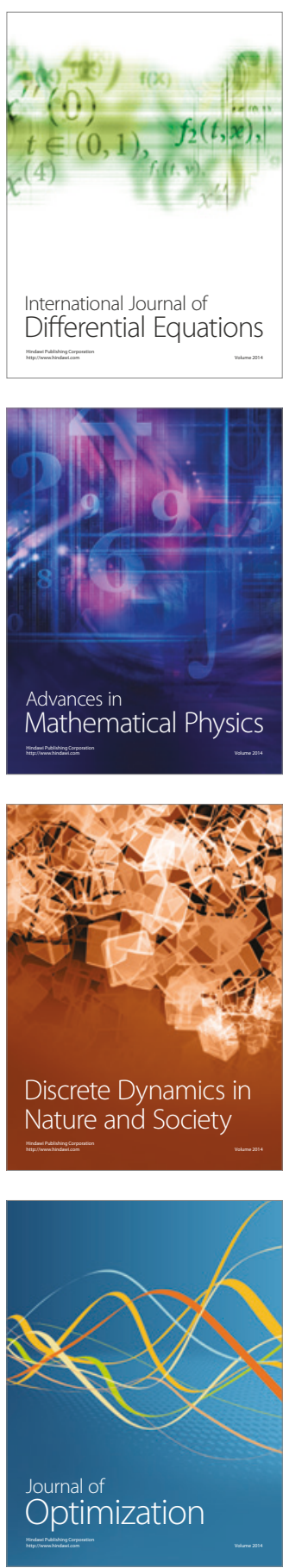